\title{
Experimental Study on Fluid Flow in Porous Media by MRI Technique Jing-Nan ZHANG ${ }^{1}$, Qin-Feng $\mathrm{DI}^{1, \mathrm{a}}$, Feng $\mathrm{YE}^{1}$, Shuai HUA ${ }^{1}$, Hui-Juan $\mathrm{CHEN}^{1}$ and Chun-Yuan $\mathrm{GU}^{1,2}$
}

${ }^{1}$ Shanghai Institute of Applied Mathematics and Mechanics, Shanghai University, Shanghai 200072, China

${ }^{2}$ Shanghai Key Laboratory of Mechanics in Energy Engineering, Shanghai 200072, China aqinfengd@sina.com

Keywords: Core flood experiment, Porous media, MRI, Displacement front, Density difference.

\begin{abstract}
Core flood experiment is commonly used to study the fluid seepage law in porous media for enhanced oil recovery, but the characteristics of fluid flow are hard to be visually described. In this paper, an advanced visual displacement system was developed and optimized to observe the shapes of flow front in the porous media. Interestingly, it is found that the flow front shows the triangular shape which can deteriorate the oil recovery. In response to this phenomenon, a series of visually core flood experiments were conducted to study the formation reasons and influencing factors of the triangular shape. Experimental results show that the density difference between the displacing and the displaced fluid is the direct factor which causes the triangular shape of the displacement front. This result suggests that the density difference between the displacing and the displaced fluid should be taken into account during oil production as well as experimental study.
\end{abstract}

\section{Introduction}

Core flood experiment was widely applied in the reservoir sensitivity evaluation[1,2], fracturing fluid evaluation[3-5],enhance oil recovery technology research[6-11], etc. However, the fluid distribution in the core can't be directly observed by means of the core flood experiment due to the core is non-transparent porous medium material. Conventional analysis method treats the core as a "black box" and predicts the fluid flow characteristics in the core by testing the pressure, flow rate, etc. In order to visually reflect the characteristics of the fluid flow in the core, many scholars had struggled to find visualization methods. Darwishet al.[12]developed a visual core-flood experiment device, in which the entry point of core holder was transparent and the camera was placed in front of it. But this visualization device could only observe the phenomenon occurring at the entry face, not within the core. Sun et al.[13]made a two-dimensional physical model of micro-layered sandstone and observed the characteristics of fluid flow through physic model by micro photography. However, this physical model could not simulate the state in three-dimensional environment, so these results had a relatively low credibility. In a word, these methods could not completely reflect the fluid distribution in the core, although it made a great contribution to the oil industry.

Fortunately, Lauterbur[14]presented a magnetic resonance imaging (MRI)principle based on nuclear magnetic resonance (NMR). The emergence of MRI technique makes it possible to observe the image of the core although initially the MRI technique was mainly used in medical field. Until recent years, magnetic resonance imaging (MRI) technique has made great progress in porous medium testing. Paulsen et al.[15] presented a MRI method utilizing paramagnetic tagging in combination with a carefully controlled and ideal flow system, it can quantitatively characterize the effects of geometry and intrinsic flow properties for a point injection into a core. Liuet al.[16]obtained a series of images of glass beads pack by MRI technique and the porosity of the sample was calculated by analyzing the image intensity. The results were in good accordance with the data obtained by the traditional method. MRI technique was proved to be a novel and effective method for measuring the porosity of porous media. Langet al.[17]established the methods to analysis the formation structure, porosity distribution, water and oil distribution by MRI technique. 
The results indicated that testing rock by MRI technique not only determine porosity and permeability parameter, but also observed the distribution of porosity and permeability. Maet al.[18]set up a visualization experimental system to investigated the $\mathrm{CO}_{2}$ migration mechanism during the displacement of supercritical $\mathrm{CO}_{2}$ and water inside the core. The porosity was measured accurately using NMR technique. Furthermore, the fraction of effective porosity and movable fluid was calculated according to the $T_{2}$ curve from NMR. Sham et al.[19]probed the structure of porous media using MRI via a combination of quantitative one-dimensional profiles and three-dimensional images, applied before and after the formation of calcite in order to characterize the spatial distribution of the precipitate. Precipitation was successfully observed at the inlet of the bead pack, whereas precipitation occurred almost uniformly along the sandstone core. Mitchell et al.[20]demonstrated the capability of low-field MRI to continuously monitor oil saturation distribution by obtaining a series of spatially resolved transverse relaxation time distributions. In author's group, Cheng et al.[21]observed successfully the fluid distribution in the core by MRI technique and the end effect in many mechanics experiments was found to exist also in natural core flow test.

The shapes of displacement front are an ever growing topic of modern day research due to it can affect the oil recovery. Spontaneous imbibition of brine into fully oil-saturated cylindrical cores was monitored by Fernøet al.[22] with MRI devices, and the displacement of refined oil with either one end open or two ends open to flow was observed as well. They found the brine invasion started at localized points and grew hemi-spherically outwards. In this paper, an advanced visualized technique was applied to observe and record the shapes of displacement front in the process of flooding, and a series of experiments were conducted to study the influence of different factors on the characteristics of the displacement front.

\section{Experimental Study}

\section{Experimental Setup}

The whole flooding process are conducted and monitored by the nuclear magnetic displacement apparatus which consists of a MINI-MRI device, a core holding unit, a measurement unit and pumps (Figure 1).In the experimental process, the core holder is placed in the MINI-MRI device which can obtain the high quality images of fluid distribution in the core. Displacing fluid is placed in three containers and three pumps provide flooding pressure, confining pressure and back pressure respectively. Fluid flows through the core under the action of displacement pressure and the inlet/outlet pressure during displacing process can be recorded to help evaluating the flow condition. Meanwhile, the images of fluid distribution in the core are captured by MINI-MRI device.

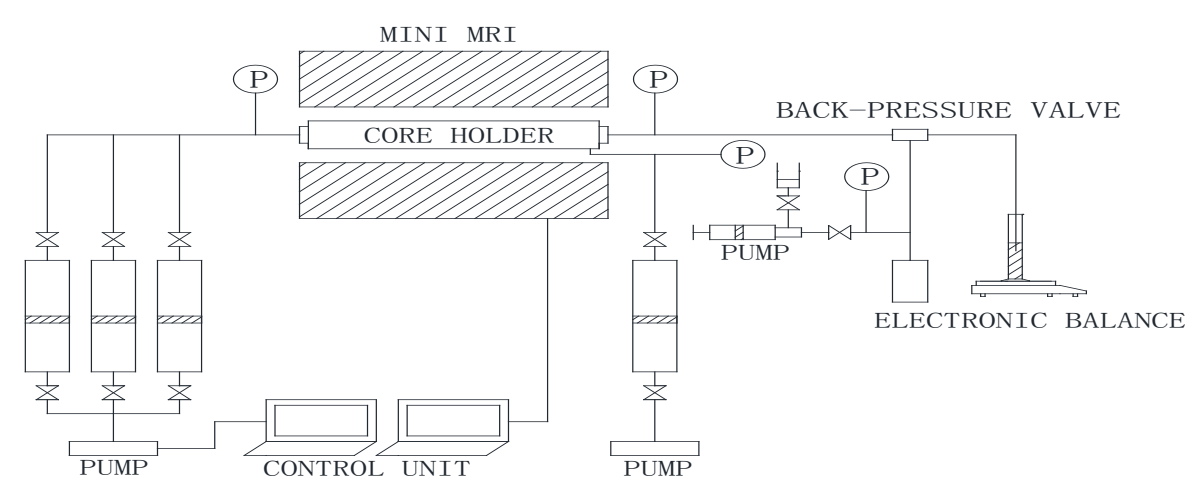

Fig. 1. Schematic diagram of nuclear magnetic resonance displacement device

\section{Experimental Method}

To observe the displacement front shape, four displacement processes were conducted, which include injecting water into the dry core, displacing heavy water with water, displacing water with 
manganese chloride aqueous solution and displacing water with heavy water. The sagittal plane images were captured by MINI-MRI device in every displacement process. In addition, three flooding scenarios were applied to study the characteristics and influence factors of the displacement front shapes. First, 0.3 pore volume (PV) of water was injected into the core and then another $0.5 \mathrm{PV}$ of water was injected into it after the core was rotated for $180^{\circ}$ (Figure 2 ). Second, water was displaced by seven different densities of manganese chloride aqueous solution. Third, manganese chloride aqueous solution was displaced by the polymer aqueous solution and water. Moreover, three different back pressure of $0 \mathrm{MPa}, 2 \mathrm{MPa}$ and $4 \mathrm{MPawere}$ selected in each displacement process.

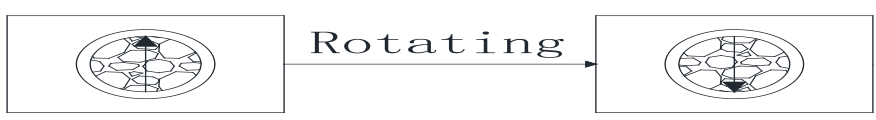

Fig. 2. Diagram of rotating the core for $180^{\circ}$

\section{Experimental Materials}

In this experiment, the manganese chloride was used to distinguish the nuclear magnetic signal of manganese chloride aqueous solution and water because the manganese chloride can inhibit the relaxation time of water in the magnetic field. Moreover, manganese chloride aqueous solutions have different density when their concentrations are different, which contribute to study the effect of density on the fluid distribution in the core. Similarly, Polymer (relative molecular mass $=2500$ ) was also applied to study the effect of viscosity on the fluid distribution in the core. In order to capture the images of the fluid distribution clearly, the ferromagnetic materials was not permitted to exist in the core, so the nonmagnetic artificial core A-1 was selected. Its properties are listed in table 1.

Table 1. Properties of core A-1

\begin{tabular}{|l|l|l|l|l|}
\hline Core number & Diameter $(\mathrm{cm})$ & Length $(\mathrm{cm})$ & Porosity $(\%)$ & Permeability $(\mathrm{mD})$ \\
\hline A-1 & 2.50 & 9.00 & 24 & 1600 \\
\hline
\end{tabular}

\section{Results and Discussions}

\section{Triangle Distribution Phenomena of the Displacement Front}

As shown in Figure 3, the displacement front distribution can be found successfully by low field MRI technique in the experiments. There are four images represented the cases of displacing air with water(Figure 3a),displacing water with manganese chloride aqueous solution(Figure 3b)displacing heavy water with water(Figure 3c), and displacing water with heavy water(Figure 3d).Interestingly, the triangular shape of the displacement front occurred. The lower triangular shapes formed when the air is displaced by water in the dry core, water is displaced by manganese chloride aqueous solution and water is displaced by heavy water. The angle between the front and the bottom of displacing agent is about 50 degree (Figure 3a), 38 degree (Figure 3b) and 36 degree (Figure 3c), respectively. However, when the heavy water is displaced by the water, the upper triangular shapes appeared and the angle between the front and bottom of displacing agent is about 144 degree (Figure 3d). 


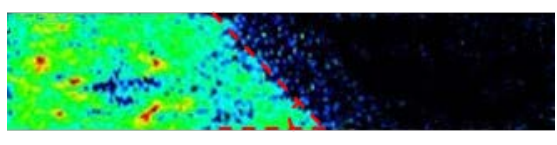

a. Water was injected into the dry core

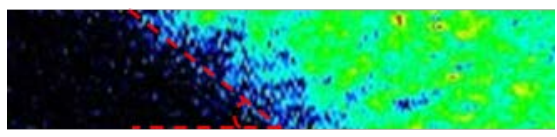

c. Displacing water with heavy water

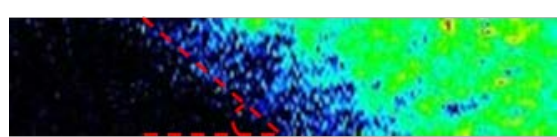

b. Displacing water with manganese chloride aqueous solution

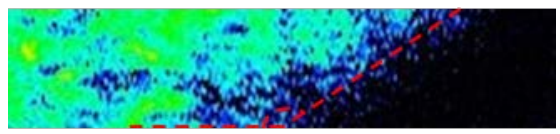

d. Displacing heavy water with water

Fig. 3. Displacement front lower/upper triangular distribution phenomenon (flooding direction $\rightarrow$ )

\section{Effect of Core Vertical Heterogeneity}

According to the reservoir rhythm characteristics, the core vertical heterogeneity may contribute to the triangle shape of fluid front in the core. For example, if the core porosity and permeability are larger in the lower part, the fluid would flow easily and vice versa. So an experiment was conducted firstly to study the effect of core vertical heterogeneity and the results are listed in Figure 4, in which five images represented five experiment steps. The image shown in step 1is obtained by MRI technique when $0.3 \mathrm{PV}$ of water was injected into the dry core, and the triangular shape can be observed clearly at the displacement front. In order to explore whether the shape of the displacement front changes over time, the filling valve was shut off and the magnetic resonance image is shown in step 2 after 12 hours. The image shows that the shape of flow front in the core has no obvious changed over time. Remarkably, the triangle shape disappeared when the core is rotated for $180^{\circ}$ along the axial direction (the image is shown in step 3 ) and there is still no obvious change (the image is shown in step 4) after keeping the filling valve shut off for 12 hours. However, the triangle shape appeared again when 0.8 PV of water was injected (as shown in step 5).

\begin{tabular}{|c|c|c|}
\hline Procedure & State & $\begin{array}{c}\text { Sagittal plane image } \\
\text { (flooding direction } \rightarrow \text { ) }\end{array}$ \\
\hline Step 1 & Water flooding for 0.3 pore volume & \\
\hline Step 2 & Shutting off the filling valve and waiting for 12 hours & \\
\hline Step 3 & Rotating the core for $180^{\circ}$ & \\
\hline Step 4 & Shutting off the filling valve and waiting for 12 hours & \\
\hline Step 5 & Water flooding for 0.8 pore volume & \\
\hline
\end{tabular}

Fig. 4. Images of the flow front shape when the dry core was flooded with water (flooding velocity of $0.5 \mathrm{ml} / \mathrm{min}$, back pressure of $0 \mathrm{MPa}$ )

These phenomena indicate that the triangular shape of displacement front has no direct correlation with the vertical heterogeneity of the core. In the whole process of injecting water, the effect of the gravity on the fluid distribution is significant, so the gravity factor is taken into consideration in the following experiments.

\section{Effect of Fluid Density}

In order to study the effect of fluid density on the shape of the displacement front, the manganese chloride aqueous solutions with different relative densities were prepared. The images of fluid in the core were captured by MRI technique when the water was displaced by manganese chloride aqueous solution (Figure 5).

According to Figure 5, the shapes of displacement front change gradually from the finger shape 
to triangular shape when the relative density of manganese chloride aqueous solution increases. The displacement fronts are finger-shaped and the outlines of it are similar to the parabola shape when the relative density of displacement fluid is between 1.01 and 1.05.In addition, the vertex of parabola moves down gradually while the relative density increases. It reaches to the bottom of core when the relative density is 1.05 , and the displacement front turns to triangular shape when the relative density is greater than or equal to 1.06.Additionally, the triangle becomes sharper with increasing the relative density of displacing fluid.

The above results show that the relative density of manganese chloride aqueous solution is the immediate cause of the triangular shape. The triangular shape of the displacement front appears if the density difference between the displacing and displaced fluid is greater than the critical value (the value is 1.06 in this study).

\begin{tabular}{|c|c|}
\hline The relative density of displacing fluid & Sagittal plane images(flooding direction $\rightarrow$ ) \\
\hline 1.01 & \\
\hline 1.02 & \\
\hline 1.03 & \\
\hline 1.04 & \\
\hline 1.05 & \\
\hline 1.06 & \\
\hline 1.07 & \\
\hline
\end{tabular}

Fig. 5. Images of the displacement front shape when the water was displaced by manganese chloride aqueous solution (flooding velocity of $0.5 \mathrm{ml} / \mathrm{min}$, back pressure of $0 \mathrm{MPa}$ )

\section{Effect of the Injected Fluid Viscosity}

In order to study the effect of the injected fluid viscosity on the displacement front shape, the water $\left(1 \mathrm{mPa} . \mathrm{s}, 20^{\circ} \mathrm{C}\right)$ and polymer $\left(5 \mathrm{mPa} . \mathrm{s}, 20^{\circ} \mathrm{C}\right)$ were prepared and used to displace manganese chloride aqueous solution respectively. The shapes of the displacement front are shown in Figure 6, in which the images are listed in two columns when the manganese chloride aqueous solution was displaced by water and polymer respectively.

\begin{tabular}{|c|c|c|}
\hline \multirow{2}{*}{$\begin{array}{l}\text { Injected } \\
\text { volume }\end{array}$} & $\begin{array}{l}\text { Manganese chloride aqueous solution } \\
\left(1 \mathrm{mPa} . \mathrm{s}, 20^{\circ} \mathrm{C}\right) \text { was displaced by water }\end{array}$ & $\begin{array}{c}\text { Polymer aqueous solution ( } 5 \\
\mathrm{mPa} . \mathrm{s}, 20^{\circ} \mathrm{C} \text { ) was displaced by water }\end{array}$ \\
\hline & \multicolumn{2}{|c|}{ (flooding direction $\rightarrow$ ) } \\
\hline $0.2 \mathrm{PV}$ & $2 y$ & 40 \\
\hline $0.4 \mathrm{PV}$ & $x_{i}$ & 5 \\
\hline $0.6 \mathrm{PV}$ & 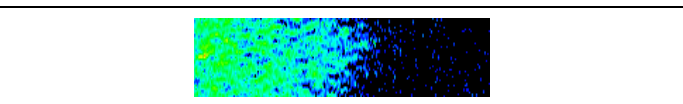 & \\
\hline $0.8 \mathrm{PV}$ & & \\
\hline
\end{tabular}

Fig. 6. Images of the displacement front shape when the flooding velocity is $0.5 \mathrm{ml} / \mathrm{min}$ 
The images show the displacement front shape had no evidence different in the process of displacing the manganese chloride aqueous solution with the water and polymer aqueous solution respectively. However, viscous fingering phenomenon appeared when the polymer was displaced by manganese chloride aqueous solution, as shown in Figure 7. This is consistent with the previous research results that viscous fingering occurs when a less viscous fluid is injected and displacing a more viscous one but the interface is stable and no finger patterns formed in the inverse situation[23,24].This result is demonstrated once again and the images of viscous fingering phenomenon in the core are also captured by the MRI technique.

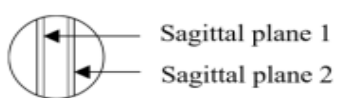

a

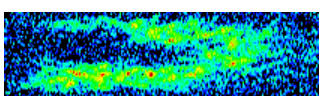

b

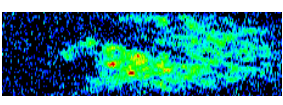

C

Fig. 7. Viscous fingering phenomenon when the polymer is displaced by manganese chloride aqueous solution(a. Sagittal plane position; b. Sagittal plane 1;c. Sagittal plane 2)

\section{Effect of Displacing Velocity}

Figure 8 shows the images of fluid distribution when the displacing velocity is $0.5 \mathrm{ml} / \mathrm{min}$ and $2 \mathrm{ml} / \mathrm{min}$, respectively.

\begin{tabular}{|c|c|c|}
\hline Injected volume & Flooding velocity of $0.5 \mathrm{ml} / \mathrm{min}$ & Flooding velocity of $2 \mathrm{ml} / \mathrm{min}$ \\
\hline $0.2 \mathrm{PV}$ & \multicolumn{2}{|c|}{ (flooding direction $\rightarrow$ ) } \\
\hline $0.4 \mathrm{PV}$ & -3 & \\
\hline $0.6 \mathrm{PV}$ & & \\
\hline $0.8 \mathrm{PV}$ & & \\
\hline
\end{tabular}

Fig. 8. Images of displacement front shape when the manganese chloride aqueous solution is displaced by water

The images indicate the upper triangle shape of displacement front is clearly visible when the manganese chloride aqueous solution is displaced by water. Moreover, the angle of flow front becomes sharper when the flooding velocity increases from $0.5 \mathrm{ml} / \mathrm{min}$ to $2 \mathrm{ml} / \mathrm{min}$. Therefore, the flooding velocity is one of the factors influencing the trend of triangle shape.

\section{Effect of back pressure}

Four different kinds of displacement were applied under the back pressure of $0 \mathrm{MPa}, 2 \mathrm{MPa}$, and 4 $\mathrm{MPa}$, respectively. The images captured at different time are shown in Figure 9. 


\begin{tabular}{|c|c|c|c|}
\hline \multirow{2}{*}{ Scenarios } & \multicolumn{3}{|c|}{ Back pressure } \\
\cline { 2 - 4 } & $0 \mathrm{MPa}$ & $2 \mathrm{MPa}$ & $4 \mathrm{MPa}$ \\
\hline $\begin{array}{c}\text { Manganese chloride aqueous } \\
\text { solution is displaced by water }\end{array}$ & & & \\
\hline $\begin{array}{c}\text { Deuterated water is displaced } \\
\text { by water }\end{array}$ & -3 & & \\
\hline $\begin{array}{c}\text { Manganese chloride aqueous } \\
\text { solution is displaced by } \\
\text { polymer }\end{array}$ & -3 & -3 & -2 \\
\hline
\end{tabular}

Fig. 9. Images of displacement front shape under the different back pressure (flooding direction $\rightarrow$ )

The images show the triangle shape appears apparently under different back pressures and it generally remain unchanged. That is, the back pressure doesn't significantly affect the shape of displacement front.

\section{Conclusions}

Experiments are conducted in this study to investigate the shape feature and influencing factors of displacement front. The following conclusions can be drawn:

(1) The triangular distribution phenomenon of displacement front is firstly observed by MRI technique. And the effects of the core vertical heterogeneity, displacement velocity, fluid density and viscosity on the shape of displacement front were studied.

(2) It is the density difference between the displacing and the displaced fluid who caused the triangular shape at the displacement front. There is a critical density difference, and if the actual density difference is larger than the critical value, the triangular shape of the displacement front will appear. In addition, the displacement velocity can affect the trend of the triangle.

(3) In order to avoid triangle shape at displacement front, the reasonable density and velocity of the injected fluid should be taken into account during oil production as well as experimental study.

\section{Acknowledgments}

This research is partly supported by the National Science Funding of China (50874071, 51274136), the Chinese National Programs for High Technology Research and Development (2008AA06Z201), the Key Program of Science and Technology Commission of Shanghai Municipality (071605102), Shanghai Program for Innovative Research Team in Universities, Shanghai Leading Academic Discipline Project (S30106) and the Shanghai Municipal Education Commission (Peak Discipline Construction Program).

\section{References}

1. Jianxiang S, Laiju H, Jianghong J. Adv. Pet. Exolor. Dev.10,38(2015)

2. Ghadami N, Das AK, Tunio KH, Sabzabadi A. International Petroleum Technology Conference (SPE, Qatar,2015)

3. Prakash C, Achalpurkar M, Uppuluri R. SPE Middle East Oil \& Gas Show and Conference(SPE,Bahrain, 2015)

4. Corrin E, Rodriguez C, Williams TM. CORROSION 2015 (NACE International, Dallas, 2015)

5. Williams NJ, Kelly PA, Berard KG.SPE International Symposium and Exhibition on Formation Damage Control (Society of Petroleum Engineers, lafayette, 2012)

6. Lei L, Guanglun L, Chuanjin Y, et al. STAE, 23,52(2013) 
7. Meixia L. Petroluem\&Petrochemical Today(Oriprobe information services, Beijing, 2008)

8. Liu G, Jiang H, Wang M. IOR 2015-18th European Symposium on Improved Oil Recovery (IOR in Challenging Environments, Tulsa, 2015)

9. Ogolo N, Olafuyi O, Onyekonwu M. SPE Saudi Arabia Section Technical Symposium and Exhibition (Society of Petroleum Engineers, AlKhobar,2012:)

10. Shaker Shiran B, Skauge A. Energy \& Fuels.27,1223(2013)

11. Dai C, Wang K, Liu Y. Energy \& Fuels.29,2304(2015)

12. Darwish MI, van Boven P, Hensens HC. SPE annual technical conference (Society of Petroleum Engineers, Houston,1999).

13. WeiSun, Zhihao Qu, Guoqing Tang.SPE International Thermal Operations and Heavy Oil Symposium and Western Regional Meeting (Society of Petroleum Engineers, Bakersfield,2004)

14. Lauterbur PC. Nature.242,190(1973)

15. Paulsen JL, Donaldson MH, Betancourt SS. J. Magn. Reson.212,133(2011)

16. Yu Liu, Yongchen Song, Jiafei Zhao. J. Test. Measure.25,195(2011)

17. Dongjiang Lang, Chengyuan Lü, Zengmen Lun. CT. Theor. Appl.21,27(2012)

18. Jin M, Rui-Na X, Shu L. J. Eng. Thermophys.33,1971(2012)

19. Sham E, Mantle MD, Mitchell J. J. Contam. Hydrol.152,35(2013)

20. Mitchell J, Staniland J, Chassagne R. J. Pet. Sci. Eng.108,14(2013)

21. Yichong Cheng, Qinfeng Di, Chunyuan Gu.J.Hydrod, Ser B.27,187(2015)

22. Fernø MA, Haugen Å, Wickramathilaka S.J. Pet. Sci. Eng.101,11(2013)

23. Mather W, Mondragón-Palomino O, Danino T. Phys. Rev. Lett.104,208101(2010)

24. Pramanik S, De Wit A, Mishra M. J. Fluid Mech.782,10(2015) 